\title{
New Dermoscopic Keys for Circumscribed Acral Hypokeratosis: Report of Four Cases
}

\author{
Paula Majluf-Cáceres ${ }^{1}$, Cristián Vera-Kellet ${ }^{1}$, Sergio González-Bombardiere ${ }^{2}$
}

1 Department of Dermatology, Pontificia Universidad Católica de Chile

2 Department of Dermatopathology, Pontificia Universidad Católica de Chile

Key words: acral hypokeratosis, image enhancement, dermatopathology, dermoscopy

Citation: Majluf-Cáceres P, Vera-Kellet C, González-Bombardiere S. New dermoscopic keyes for circumscribed acral hypokeratosis: report of four cases. Dermatol Pract Concept. 2021;11(2):e2021010. DOI: https://doi.org/10.5826/dpc.1102a10

Accepted: August 9, 2020; Published: March 8, 2021

Copyright: $@ 2021$ Majluf-Cáceres et al. This is an open-access article distributed under the terms of the Creative Commons Attribution License BY-NC-4.0, which permits unrestricted noncommercial use, distribution, and reproduction in any medium, provided the original author and source are credited.

Funding: None.

Competing interests: The authors have no conflicts of interest to disclose.

Authorship: All authors have contributed significantly to this publication.

Corresponding author: Paula Majluf-Cáceres, MD, Centro Médico San Joaquin, Vicuña Mackenna 4686, Macul, Chile. Email: pamajluf@ uc.cl

\section{Introduction}

Descriptions of the dermoscopic features of acral hypokeratosis $(\mathrm{AH})$ are few. Clinically it can resemble other entities, such as Bowen disease or porokeratosis of Mibelli. Although $\mathrm{AH}$ is considered a benign pathology, in 2010 a case with actinic keratosis in the hypokeratosic epidermis and underlying elastosis was reported [1], hence the importance of knowing the dermoscopic findings for an early diagnosis and to rule out other differential or coexisting diagnoses.

\section{Case Presentation}

Our case series was comprised of 4 patients with AH confirmed by biopsy in the hypothenar eminence. Figure $1 \mathrm{~A}$ shows $\mathrm{AH}$ in a 61-year-old and Figure $1 \mathrm{~B}$ a 78 -year-old woman with a 10 -year history of AH. Dermoscopy revealed pink areas on a red milky blush with scattered red dots, steplike scales at the periphery, and elongated whitish structures in a fibrillar raindrop pattern (Figure 1, C and D).

The third case corresponded to asymptomatic AH (Figure 1E) that had developed 2 weeks after a cutting wound in a 30-year-old woman. Dermoscopy showed a red dot pattern over a homogeneous red-yellow area (Figure $1 \mathrm{~F}$ ). The fourth case was a 54-year-old woman affected by AH for 8 years (Figure 1G). Dermoscopy revealed a fine white pseudonetwork, pink stiff areas on a red milky blush with red dots, step-like scales at the periphery, and elongated whitish structures in a fibrillar raindrop pattern. (Figure 1H). Microscopy revealed an area of hypokeratosis demarcated by a sharp and frayed cut-off from uninvolved acral skin with discrete hypogranulosis, dilated blood vessels in the papillary dermis, and slightly thickened collagen fibers in the reticular dermis (Figure 2). 


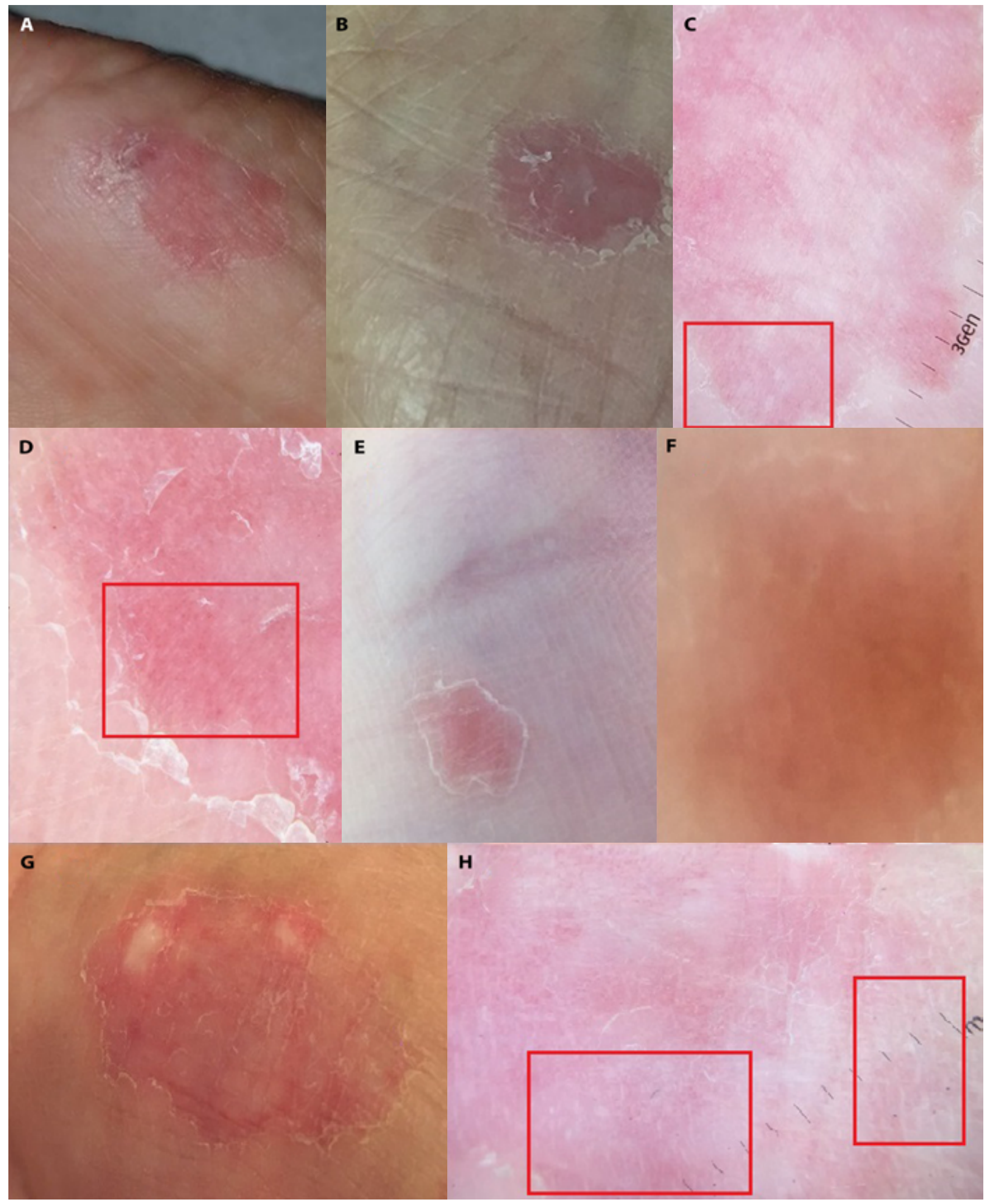

Figure 1. Clinical and dermoscopic features. (A and B) Cases 1 and 2: Atrophic erythematous plaque with an irregular hyperkeratotic border. (C) Dermoscopy shows pink areas on a red milky blush with scattered red dots, step-like scales at the periphery and elongated whitish structures in a raindrop pattern. (D) Dermoscopy shows red milky blush, pink islets with dotted vessels, elongated whitish structures in raindrop pattern and staircase sign. (E) Case 3: Depressed erythematous plaque, surrounded by an hyperkeratotic border. (F) Dermoscopy showed a red dot pattern over a homogeneous red-yellow area. 


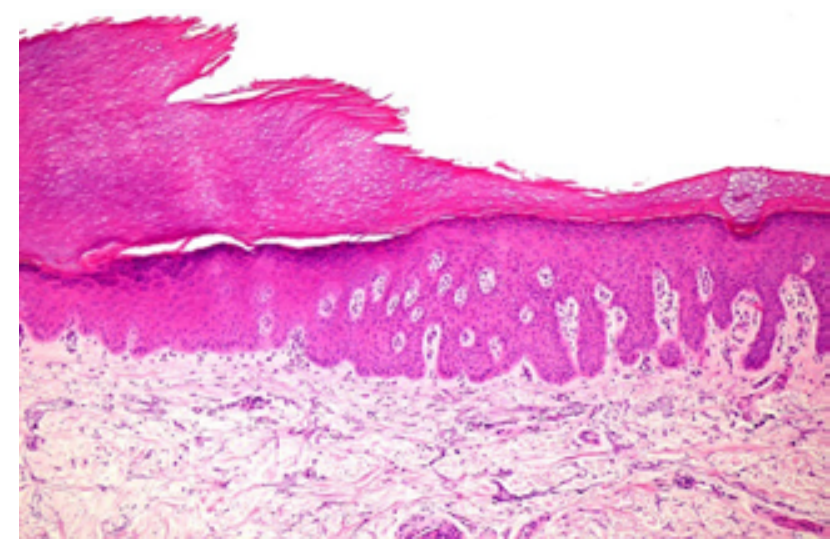

Figure 2. Histology displays an area of hypokeratosis demarcated by a sharp and frayed cut-off from uninvolved acral skin with discrete hypogranulosis, dilated blood vessels in the papillary dermis and slightly thickened collagen fibers in the reticular dermis (H\&E, $\times 10)$.

\section{Conclusions}

Previous case series reported star-like desquamation at the periphery,and a well-demarcated erythema with reddish dots. These structures correlate with histopathological studies showing a sharply demarcated area of hypokeratosis, dilated capillaries in the papillary dermis and vessels in the upper reticular dermis [2]. A recent case report of congenital plantar $\mathrm{AH}$ showed a white thin scale and a reticulated surface with no visible acrosyringia opposing the typical dermoscopic acral pattern [3].

Our study revealed different dermoscopic findings than previously published: A fine white pseudonetwork and elongated whitish structures in a "raindrop pattern" found in those patients with longstanding $\mathrm{AH}$ and could be correlated with the increasing collagen proliferation and thickening. Thus in our case of 2-weeks' onset AH, only a yellowish-red blush and red dots with peripheral step-like scales were distinguishing.

\section{References}

1. Kanitakis J, Lora V, Balme B, Roby J. Premalignant circumscribedpalmar hypokeratosis: a new form of circumscribed palmar hypokeratosis? Case report and literature review. Dermatology. 2010;220(2):143-146. DOI: 10.1159/000264607. PMID:19955702.

2. Nishimura M, Nishie W, Nakazato S, Nemoto-Hasabe I, Fujita Y, Simizu H. Circumscribed palmar hypokeratosis: correlation between histopathological patterns and dermoscopic findings. Br J Dermatol. 2012;167:221-222. DOI: 10.1111/j.13652133.2012.10855.x. PMID:22283364.

3. Bassi A, Oranges T, Massi D, Piccolo V, Mazzatenta C, Neri I. Congenital circumscribed plantar hypokeratosis. Int J Dermatol. 202059(10):e367-e369. DOI: 10.1111/ijd.14967. PMID: 32516442 . 